ArtefaCToS. Revista de estudios de la ciencia y la tecnología

eISSN: $1989-3612$

Vol. 10, No. 1 (2021), 2. ${ }^{\text {a }}$ Época, 107-120

DOI: https://doi.org/10.14201/art2021101107120

\title{
Las ciencias sociales e ingenieriles desde el realismo constructivo de Ronald Giere
}

\author{
Social and Engineering Sciences from Ronald Giere Constructive \\ Realism
}

\author{
Ana CUEVAS BADALLO*; Obdulia TORRES GONZÁLEZ ${ }^{* *}$ \\ *Universidad de Salamanca, España \\ acuevas@usal.es \\ (D) https://orcid.org/0000-0001-8080-4233 \\ ${ }^{* *}$ Universidad de Salamanca, España \\ omtorres@usal.es \\ (D) https://orcid.org/0000-0003-1620-6911
}

Recibido: 03/03/2021. Revisado: 10/04/2021. Aceptado: 13/04/2021

\section{Resumen}

El realismo constructivo defendido por Ronald Giere puede servir para comprender mejor las características de dos ámbitos de la investigación científica que tienden a ser menospreciados por las concepciones filosóficas más tradicionales: el conocimiento social e ingenieril. Aquí se defenderá que estos dos ámbitos comparten rasgos relevantes y que la propuesta modelística del autor puede servir para llevar a cabo un mejor análisis de sus peculiaridades. Enfatizaremos, por un lado, su concepción modelística de la ciencia, y la consideración de que los modelos guardan relaciones de similitud con el mundo, y por otro cómo esta postura incide en la elección entre teorías. En concreto, se tratará acerca de la naturaleza especial de las leyes en ciencias sociales y en las ciencias ingenieriles, así como los criterios por los que los científicos en estos ámbitos eligen entre los distintos modelos posibles.

Palabras clave: elección de modelos; leyes ceteris paribus; relación de similaridad. 


\begin{abstract}
The constructive realism upholded by Ronald Giere can be used for a better understanding of the features of two fields of scientific research that tend to be demeaned by the traditional philosophical conceptions: social and engineering knowledge. Here we will argue that these two areas share relevant features, and that Giere's modelistic proposal can be used to carry out a better analysis of their peculiarities. We will emphasize, on the one hand, his modelistic conception of science, and his consideration that models are similar to the world, and on the other, how this position affects the choice between theories. Specifically, we will deal with the special nature of laws in the social and engineering sciences, as well as the criteria by which scientists in these fields choose between different possible models.
\end{abstract}

Keywords: model election; ceteris paribus laws; similarity relation.

\title{
1. Introducción
}

Ronald Giere nunca se dedicó de forma explícita a la reflexión sobre las ciencias ingenieriles o las ciencias sociales. Sin embargo, su realismo constructivo, una postura filosófica que quería poner de relieve el aspecto social de la construcción del conocimiento científico sin que ello supusiera un menoscabo para el valor de este, tiene un buen encaje en las reflexiones sobre el conocimiento social e ingenieril. Desde nuestro punto de vista, estos dos ámbitos comparten algunos rasgos relevantes y creemos que la propuesta modelística del autor puede ser altamente pertinente para su análisis.

En este artículo abordaremos el realismo constructivo de Giere enfatizando, por un lado, su concepción modelística de la ciencia, según la cual los modelos no establecen una relación de correspondencia ni isomorfismo con el mundo, sino de similitud; y por otro cómo esta postura incide en la elección entre teorías. Este realismo constructivo fue sumamente controvertido debido fundamentalmente a su defensa de un realismo sin verdad. Fue uno de los primeros autores que consideró que la ciencia se asemejaba mucho a la tarea de confeccionar mapas que nos habrían de servir para guiarnos en nuestro transitar por el mundo. Una idea muy sugerente del conocimiento científico que llevó hasta sus ultimas consecuencias cuando incorporo la noción perspectivista, porque un mapa siempre es un mapa para alguien y para algo, es decir, tiene una finalidad específica para el que lo usa, y será bueno o malo dependiendo de para qué lo queramos. Esta idea de finalidad de los modelos y de los valores para elegirlos resulta sumamente productiva en el ámbito de la reflexión acerca de lo social y lo ingenieril. Por ello, en este artículo nos hemos centrado en la reflexión en torno a dos cuestiones fundamentales. La primera es la naturaleza de las leyes. El hecho de que todas las leyes posean cláusulas ceteris paribus sitúa a todos los ámbitos científicos en pie de igualdad en lo que a cientificidad se refiere. Poseer leyes universales y sin excepción, que había 
ha sido el marchamo que confería genuina cientificidad en las concepciones más tradicionales, pierde su validez en favor de una noción mucho más modesta de las leyes, común para todos los ámbitos y no solo para las ciencias sociales y las ciencias ingenieriles.

La segunda de las cuestiones que abordaremos tiene que ver con los criterios por los que los científicos eligen entre los distintos modelos posibles. Giere incorporó los motivos valorativos como parte del proceso de elección entre teorías, sin que ello supusiera una incursión en el ámbito subjetivista. Esta perspectiva axiológica es sumamente útil, cuando nos enfrentamos a ámbitos de conocimiento que tienen una vertiente de intervención en el mundo, una intervención que tiene que ser eficiente y segura, y además, cuidadosa con sus posibles impactos sociales.

\section{El realismo constructivo}

Ronald Giere forma parte de un conjunto de filósofos de la ciencia posteriores al giro historicista, que han querido, por un lado, hacer un análisis de la ciencia que tuviese en cuenta aspectos que la Concepción heredada había pasado por alto, pero que a la vez intentase superar los embates de las concepciones más radicalmente constructivistas y antirrealistas. Giere propuso en su lugar un "realismo constructivo", una versión naturalizada de la filosofía de la ciencia, que empleará la ciencia cognitiva y sus herramientas para caracterizar el proceso creativo e investigador. Según Giere, los científicos son agentes con determinadas capacidades cognitivas, de manera que, si queremos comprender su trabajo deberemos tener en cuenta lo que nos dice la psicología.

Giere defiende que la ciencia es una práctica situada. Considerar a la ciencia como una representación pasiva del mundo o como una pura forma lógica es, según él, una concepción equivocada. Asimismo, enfatiza el aspecto colectivo de la investigación científica, la pluralidad de variables culturales y disciplinarias presentes, tanto en las estrategias de investigación como en los aspectos evaluativos, y las normas que gobiernan las actividades comunitarias, prestando menos atención a la cognición individual o los sistemas abstractos de proposiciones. Su proyecto, como él ha expuesto en diversas ocasiones, ha sido construir un marco teórico para el estudio de la ciencia, que se situase a medio camino entre la filosofía de la ciencia tradicional y el nuevo constructivismo social. Eso sí, sin caer en relativismos excesivos que amenazan con convertir a la ciencia en una actividad sin ninguna excepcionalidad epistémica.

Desde esa concepción naturalizada, Giere quiere dar cuenta de dos problemas centrales a los que se enfrenta la reflexión sobre la ciencia. Uno es la naturaleza de la representación científica, esto es de las teorías científicas, y el otro es explicar los motivos por los que se elige entre diferentes teorías. 
En cuanto al primer asunto Giere afirma que las teorías científicas, en lugar de conjuntos de proposiciones verdaderas acerca del mundo, se pueden entender como conjuntos o familias de modelos o mapas cognitivos representacionales. Los seres humanos, al igual que otros muchos animales, tenemos la capacidad de generar estos mapas para representarnos el mundo y solucionar problemas. Nuestros modelos son sofisticados y suelen emplear el uso de elementos no lingüísticos (visuales) y lingüísticos (conceptuales) relacionados entre sí por vínculos cognitivos. Cada modelo o familia de modelos puede llevar asociada una hipótesis de aplicabilidad, que afirmaría que el modelo guarda cierta relación de similitud con algún sistema o tipo de sistemas existentes en la realidad. De manera que, la relación que se establece entre los modelos y la realidad de la que tratan es de similitud (y no de isomorfía como propone Van Fraassen).

Frente al empirismo constructivo de Bas Van Fraassen, la relación de semejanza entre los modelos y el sistema real no tiene por qué limitarse a sus aspectos observables. Lo importante es la semejanza entre los dos sistemas y no su correspondencia. Además, la semejanza es una cuestión de grados y de aspectos, lo que conllevaría una concepción perspectivista.

Ya no hay teorías verdaderas, sino catálogos de casos en los cuales los modelos encajan tolerablemente bien con los sistemas del mundo (Giere, 1988, 92). Estos modelos se generan usando principios generales y condiciones específicas (Giere, 2003 , 5). Los principios generales, que según otras interpretaciones son las leyes científicas, no son verdades universales, sino verdades, en todo caso, consensuadas por los científicos para el modelo en cuestión. El grado de encaje (fitness) dependerá de cuestiones pragmáticas y no solamente ontológico-epistémicas. Es decir, además de los motivos generalmente considerados por la filosofía, los científicos pueden recurrir a otro tipo de criterios que permitirán determinar si un modelo encaja o no con los sistemas del mundo. De esta manera, la relación de similitud permite superar la caracterización ingenua del realismo. Ahora, el objetivo de la empresa científica no es ofrecer una caracterización literalmente verdadera del mundo, sino simplemente producir modelos similares en ciertos aspectos con el mundo, similitud que se determina por el contexto científico.

En este sentido, su visión realista de la ciencia incorpora ciertos elementos constructivistas, habitualmente juzgados como esencialmente críticos con el realismo. Considera que la ciencia está realizada por seres humanos, lo cual no es una característica tan baladí como puede parecer. Según este autor, los científicos, que son agentes intencionales, usan los modelos para representar ciertos aspectos del mundo con determinados propósitos. En la elección del mejor modelo posible intervienen, desde el primer momento ciertas "normas", siendo la más importante la de escoger aquel modelo que proponga la mejor representación posible, aunque será la comunidad científica la que consensuará la validez de los motivos. No es necesario dejar de lado los intereses epistémicos que los científicos tienen cuando toman este tipo de decisiones. "Epistemic interests operate 
on the same level as non-epistemic interests. More importantly, my account does not require that individual scientists be able to separate out their epistemic from their non-epistemic interest”. (Giere, 1992, 102).

Otro aspecto interesante de la explicación de Giere es que su perspectiva constructivista no implica un relativismo desde el punto de vista ontológico. Los científicos escogen aquellos aspectos de los fenómenos sobre los que van a tratar, es decir, las propiedades que ellos consideran relevantes, siendo conscientes de que dejan otras muchas de lado (Giere, 1999, 180). Ello no quiere decir que se "inventen" la realidad, sino que el mundo es lo suficientemente complejo como para que se puedan proponer diferentes modelos sobre los mismos fenómenos, sin que signifique necesariamente que uno de ellos ofrece la mejor perspectiva posible. Todo depende del propósito del modelo. Y, por supuesto, el propósito puede variar contextualmente.

Hay quien ha considerado (por ejemplo Andy Pickering en "Philosophy Naturalized a Bit" y Norton Wise en "Realism is dead") que la concepción de Giere puede conducir a tesis relativistas y que no puede denominarse propiamente realista. Por un lado, al reconocer Giere la importancia de los diferentes intereses a la hora de escoger el mejor modelo posible, parece estar dejando la puerta abierta a un relativismo epistémico. Como se ha comentado antes, los modelos no pueden capturar todos los aspectos del sistema real, y tampoco existe una relación de correspondencia uno a uno entre todos los aspectos del modelo y algunos aspectos del sistema real. Con similares conclusiones, Nancy Cartwright concluye una perspectiva antirrealista. ¿Por qué Giere prefiere seguir sosteniendo que su concepción de la ciencia es realista? Él mismo lo argumenta:

My form of realism requires two things. First, that it makes sense to suppose that some models fit the world better than others in recognized aspects and to measurable degrees. Second, that it is possible to design experiments which make it more likely, although maybe still far from certain, that one will choose the better fitting model $(1992,103)$.

De manera que no es necesario abandonar el ideal axiológico de que existen modelos que representan mejor la realidad, un ideal que también se manifiesta en la noción de progreso en el conocimiento científico, algo que los constructivistas sociales no estarían dispuestos a aceptar. Giere no niega que existen contingencias y negociación social en el proceso de hacer ciencia, o que los intereses personales, profesionales o sociales tienen un gran poder, pero también afirma que las estrategias experimentales pueden sobrepasar las interacciones sociales y los intereses, dejando poca libertad a los científicos para elegir el mejor modelo.

El realismo de Giere es un realismo sin verdad o falsedad. Estas nociones son, desde su perspectiva, poco precisas para dar cuenta de la complejidad que 
supone hacer afirmaciones científicas sobre el mundo. De hecho, si muchas de esas afirmaciones científicas son tomadas literalmente podrían considerarse falsas (Giere, 2005, 290).

Entonces, ¿por qué seguir considerándose realista? Según él, se puede sostener una concepción más deflacionaria del realismo, una que acepte una visión de la naturaleza del conocimiento científico, según la cual el mundo de nuestra experiencia no es independiente de nuestras mentes, aunque el mundo en sí mismo no depende de la existencia de mentes y el mundo que es investigado por la ciencia es diferente del mundo "en sí mismo". Una concepción que no recurra a la verdad, entendida esta como verdad como correspondencia, sino a descripciones cada vez mejores del mundo podría considerarse realista, ya que no abandona sus compromisos metafísicos y epistémicos fundamentales. Ahora bien, en el caso de Giere esta perspectiva se enriquece, se acerca a la práctica científica real, admitiendo que la ciencia es un constructo social, aunque no cualquier constructo social. Por que su pretensión, como el propio Giere reconoce:

is to develop an understanding of scientific claims that mediates between the strong objectivism of most scientists, or the hard realism of many philosophers of science, and the constructivism found largely among historians and sociologists of science. My understanding will turn out to be closer to that of objectivist scientists and realist philosophers of science than to that of constructivists. Nevertheless, in spite of the excesses of some constructivists, I think there is a valid point to the constructivist critique of science. The challenge is to do justice to this point while avoiding the excesses (Giere 2006, 3).

\section{La propuesta de Giere para el caso de las ciencias ingenieriles}

La propuesta de Giere tiene la virtud de que, al prescindir de una consideración estricta de la función de representación y de verdad de las teorías científicas, se adapta mejor para comprender otro tipo de ciencias, como son las ciencias ingenieriles y las ciencias sociales. Veamos en este apartado cómo emplear esta concepción realista constructiva para el caso de las ciencias ingenieriles.

Estas podrían considerarse clusters o familias de modelos y los principales tipos de modelos empleados en estas ciencias son: (i) materiales, entre los que incluiría a los mapas, los diagramas y los modelos a escala y, (ii) los modelos abstractos, que serían los modelos matemáticos puros, los modelos matemáticos aplicados y los modelos teóricos, construidos estos últimos con principios teóricos. Todos estos modelos serán utilizados en función de los diferentes propósitos que inspiren esas investigaciones.

Los principios generales no son universales, necesarios o verdaderos, tal y como se exigía que fuesen las leyes de las ciencias. Si estos requisitos son de difícil 
cumplimiento para las leyes de las ciencias básicas, en el caso de los principios de las ciencias ingenieriles sería contraproducente exigírselos. Y ello se debe, fundamentalmente, a que los principios generales de las ciencias ingenieriles no emplean abstracciones y simplificaciones de la misma manera que se utilizan en las ciencias básicas, aceptando en todo caso, las cláusulas ceteris paribus necesarias para hacer que esos principios puedan emplearse en la predicción exitosa del comportamiento de fenómenos concretos.

De igual manera, las ciencias ingenieriles reconocen la heterogeneidad ontológica, en contra de concepciones homogeneizadoras: el mundo es excesivamente complejo como para poder ser caracterizado con un solo y único modelo. Por ello, la elección entre modelos vendrá determinada por los propósitos de los investigadores, sujetos activos según esta nueva interpretación, ya que diferentes propósitos pueden motivar la creación de distintos modelos. Lo que hacen las teorías es proponer conjuntos de modelos, que caracterizan o explican cómo se comportan determinados sistemas, que no son otra cosa más que aspectos parciales de la realidad.

\subsection{Similitud entre los modelos y el mundo}

La noción de similitud o fitness ha sido uno de los aspectos más criticados de la explicación propuesta por Giere. Por un lado, las críticas señalan que es necesario responder a la pregunta de cómo un modelo, que es un objeto abstracto, puede ser similar a un objeto concreto del mundo. Paul Teller responde a esta cuestión: la similitud se produce entre las propiedades de los modelos y las propiedades de los sistemas concretos y no entre diferentes categorías ontológicas (Teller, 2001, 399).

Pero además existe otro problema: el referente a los motivos que los científicos tienen para escoger entre diferentes modelos. Según Giere, los científicos, por lo general, eligen aquellos modelos que mejor representan los aspectos de la realidad que les interesan. Con objeto de saber hasta qué punto esto es así, se suelen llevar a cabo experimentos, cuyos resultados harán inclinar la balanza en un sentido u otro (Giere, 1999a, 53). Se puede replicar que tampoco este es un criterio válido para establecer el grado de similitud que puede aportar un modelo, ya que los fenómenos sobre los que tratan los experimentos no ocurren "naturalmente", sino que son producidos y mantenidos artificialmente en condiciones especiales en los laboratorios. Sin embargo, a pesar de que los experimentos se llevan a cabo en condiciones particulares, lo cierto es que pueden repetirse de manera controlada, por diferentes científicos en laboratorios distintos, sin que ello altere el valor de los resultados.

Los científicos, según la explicación de Giere, no toman decisiones como lo haría un agente según la explicación de Bayes. Pero tampoco se puede decir que se comporten como satisficers según la interpretación de Herbert Simon (Simon, 
1945). Si se acepta que son agentes racionales, lo son en el sentido de que su comportamiento está dirigido al logro de ciertos objetivos: así, cuanto más efectivo sea su comportamiento, más racional puede ser considerado (Giere, 1988, 160; Giere, 1999a, 82). Se trata, como puede verse, de una racionalidad "condicional" o "instrumental": los científicos se comportan de manera racional cuando escogen aquel modelo que mejor representa la realidad.

Ahora bien, este no es el único criterio empleado para escoger entre modelos, pueden existir y de hecho lo hacen, otros valores, criterios e intereses para tomar esa decisión. Asimismo, cabe preguntarse si existen diferencias en los objetivos de los ingenieros y de los científicos básicos a la hora de construir sus respectivos modelos. En el caso de las ciencias ingenieriles, el objetivo principal es proporcionar modelos teóricos y matemáticos que sean de utilidad en el diseńo de artefactos seguros, duraderos y eficientes. En el caso de las ciencias "básicas", el objetivo principal reconocido es obtener conocimiento acerca de cómo son y se comportan las cosas, esto es, proponer modelos que representen y expliquen pormenorizadamente determinados aspectos, que nos interesan acerca de la realidad. Esa diferencia de objetivos lleva aparejadas diferencias en la valoración de los atributos que se han de considerar "deseables" en los modelos.

Algunos filósofos han intentado plantear una lista de atributos deseables para las diferentes ciencias: precisión, consistencia (tanto interna como referente a otras teorías consolidadas relevantes), alcance (sus consecuencias deben ir más allá de los datos que directamente explica), simplicidad (capacidad de presentar de forma organizada fenómenos que de otra forma aparecen aislados y confusos), y fertilidad (para ulteriores investigaciones) (Kuhn, 1977); consistencia interna, predicción de resultados inesperados y variedad de evidencias (Laudan, 1990); adecuación empírica, novedad, heterogeneidad ontológica, interacción mutua, aplicabilidad a necesidades humanas presentes y difusión de poder(Longino, 1996). Sin embargo, no existe un algoritmo que automatice los juicios de esta índole, por lo que dependerán del adiestramiento individual del científico, de sus objetivos y experiencia personal. Además, los científicos no tienen porqué valorar las características deseables de la misma manera (McMullin, 1982, 16). Una teoría, una hipótesis o un modelo pueden resultar empíricamente adecuados, pero en absoluto simples, o puede darse el caso de que siendo simples no presenten un gran poder explicativo, etc. Por eso los científicos necesitan sopesar los distintos atributos y seleccionar el mejor modelo entre diferentes posibilidades.

Para hacer tal cosa se podría concebir que los científicos actúan como agentes maximizadores bayesianos o, al menos, como satisficers como sugiere Simon. Sin embargo, ambos planteamientos resultan problemáticos, como apunta Giere (1988, 145-165. Contra la aplicación de la teoría de juegos a la toma de decisiones sobre hipótesis, ver también Giere, 1969).

En el caso de las ciencias ingenieriles, el conjunto de valores, que se emplean para escoger entre diferentes modelos posibles, está influido notablemente por la 
necesidad de que estos modelos puedan ser aplicados a situaciones reales concretas, situaciones de construcción de artefactos que han de comportarse de manera segura en el mundo. Este motivo penetra en todos los demás y los determina, de tal manera que se puede llegar a sacrificar atributos, que desde el punto de vista de las ciencias básicas son insustituibles. Ello no significa que los modelos de las ciencias ingenieriles sean peores o menos verdaderos, significa, simplemente que son mejores desde el punto de vista de la aplicabilidad de los mismos.

De esta manera, la propuesta de Giere ayuda a comprender por qué pueden coexistir conjuntos de modelos aparentemente contradictorios, pero que pueden ser elegidos, debido a que reúnen mejores características para el caso concreto de aplicación del modelo.

\section{La propuesta de Giere para el caso de las ciencias sociales}

Giere nunca se dedicó de forma explícita a la reflexión sobre las ciencias sociales, aunque tampoco afirmó que estas debieran seguir otros modelos de explicación. De hecho, sus planteamientos inciden directamente en los grandes debates metateóricos de estas disciplinas.

Uno de estos grandes debates tiene que ver con la existencia de leyes científicas en ciencias sociales. Bajo una concepción tradicional las leyes son las que otorgan el marchamo de ciencia a una disciplina, ya que, bajo el modelo nomológico deductivo las leyes son absolutamente imprescindibles para los dos grandes objetivos de la empresa científica: predecir y explicar. Sin demasiada discusión, podemos afirmar que una ley es una proposición verdadera, necesaria, universal y objetiva. Estas características, ya de por si problemáticas, se vuelven aún más discutibles cuando intentamos aplicarlas al conocimiento de lo social. La naturaleza objetiva de las leyes sociales es escurridiza, dado que exige que su existencia sea independiente de su formulación por parte de los seres humanos. La exigencia de necesariedad nos conduce al problema del determinismo y el libre albedrío, pero es sobre todo el requerimiento de universalidad lo que ocasiona más problemas. La única manera de mantener la universalidad de las leyes en ciencias sociales es recurriendo a cláusulas ceteris paribus, que equivalen a condiciones antecedentes adicionales más o menos indefinidas: "si todo lo demás permanece igual", "si nada interfiere", "si no intervienen factores adicionales". Son leyes tales que puede darse la condición antecedente y no la consecuente. Por ejemplo, según una de las leyes mejor establecidas de la economía, la ley de la demanda, la cantidad que se desea adquirir por unidad de tiempo será tanto mayor cuanto menor sea el precio. Esta ley conllevaría una clausula ceteris paribus más o menos implícita que supone: una economía estable, pleno empleo, mercado libre, consumidor racional y gustos constantes. El debate así se traslada de la existencia de leyes en las ciencias sociales al carácter de ley de las leyes ceteris paribus. Aquí entra de lleno la propuesta de Giere, para quien las leyes fundamentales de la ciencia no 
son ni universales, ni necesarias, ni tan siquiera verdaderas (Giere, 1999, 90) a no ser que incluyamos una condición manifiesta, que explicite las condiciones de aplicación de la ley y elimine las idealizaciones presentes en las mismas. Esa fue la estrategia del empirismo lógico para las ciencias sociales, la especificación de la cláusula ceteris paribus (Gómez Rodríguez, 2001). Pero esta especificación convertía a las leyes sociales en enunciados sumamente complejos, donde se restringía el dominio de aplicación y por tanto la universalidad de las mismas (Gibson, 1982, 167-168).

La propuesta de Giere es distinta, para él la especificación de la cláusula convierte a las leyes en enunciados vacíos. "My objection to this interpretation is that it is impossible to fill in the proviso so as to make the resulting statement true without rendering it vacuous" (Giere, 1999, 91). Esta línea argumental, que considera que las leyes cualificadas con cláusulas ceteris paribus son enunciados vacíos, es la seguida por autores como John Roberts en el debate de la existencia de leyes en ciencias sociales. Para Roberts, no existen leyes en ciencias sociales, porque una ley cualificada con una cláusula ceteris paribus no es una ley genuina sino un enunciado vacío. Sin embargo, para el autor si existen leyes genuinas en ciencias naturales (Roberts, 2004). Sin embargo, en términos generales el enfoque de Giere supuso un respiro para los teóricos sociales, puesto que ya no tenían que defender la cientificidad de sus disciplinas. La posesión de leyes de carácter universal no era un rasgo necesario del conocimiento científico y la idealización de las mismas era una situación común a las ciencias naturales y sociales.

En el enfoque de Giere leyes y teorías se dejan de lado a favor de una interpretación basada en una concepción modelística de la empresa científica. En esta concepción:

scientists generate models using principles and specific conditions. The attempt to apply models to the world generates hypotheses about the fit of specific models to particular things in the world, hypotheses that may be generalized across previously designated classes of objects (Giere, 2004, 744).

Así tenemos principios generales, modelos e hipótesis teóricas. Los principios generales serían lo que tradicionalmente se ha denominado leyes fundamentales de las teorías, tales como las leyes de Newton, la ley de la relatividad general o el principio de selección natural. En términos de Ulises Moulines principios guia y en los de Thomas S. Kuhn generalizaciones simbólicas. En la propuesta de Giere, tal y como se ha señalado, si son referidas al mundo estas leyes serían falsas o trivialmente vacías. Su función es la de definir los modelos y no la de referirse a nada en el mundo. Giere señala como principios de este tipo explícitamente a los principios de equilibrio general de la economía neoclásica (2004, 74). Los modelos son de muy diversas clases: físicos, a escala, análogos o matemáticos por citar algunos. En ciencias sociales disponemos de este tipo de modelos y su uso 
ha caracterizado el desarrollo de las mismas a través del tiempo. De hecho, los modelos analógicos constituyen en muchas ocasiones los inicios de una teoría. Ya Hobbes caracterizaba al Estado como un gran Leviatán:

que no es sino un hombre artificial, aunque de mayor estatura y robustez que el natural para cuya protección y defensa fue instituido; y en el cual la soberanía es un alma artificial que da vida y movimiento al cuerpo entero [...] la recompensa y el castigo son los nervios que hacen lo mismo en el cuerpo natural ... la concordia es la salud; la sedición la enfermedad; la guerra civil la muerte (Hobbes, [1651] 1983, 25).

Tenemos también modelos matemáticos, como el homo economicus, regido por la maximización de la utilidad. En este caso imponemos un formalismo matemático, sobre la situación física, esperando que capte lo esencial de esa situación, la conducta humana. Tenemos incluso modelos físicos como la máquina Newlyn-Phillips, una máquina hidráulica que representa a la macroeconomía. La relación que propone Giere entre los modelos y el mundo es una relación de similaridad. Para lograr esta relación, los científicos señalan aquellos aspectos del modelo que son similares a aquellos del mundo real que han sido designados. Hay dos cuestiones sumamente importantes aquí, una es la selección de unos rasgos determinados, que tiene que ver con los conceptos de idealización o abstracción, y otra la selección de esos rasgos por parte del científico. Ya Stuart Mill, por ejemplo, señalaba que la Economía Política:

hace abstracción de todas las pasiones o motivaciones humanas, excepto aquellas que pueden considerarse como deseos antagonistas perpetuos del deseo de riquezas, es decir, la aversión al trabajo y el deseo de goce presente de costosos placeres (Mill, 1836, 321).

Es decir, el único rasgo relevante en el modelo de homo economicus de Mill es el deseo de riqueza. Por tanto, son esos rasgos los que se seleccionan para formar parte del modelo y ver en que medida se adecúan al mundo.

Esa similaridad es propuesta por la hipótesis teórica. La similaridad debe además ser especificada al menos en algún grado y señalar aquellos aspectos que son relevantes. Por ejemplo: "The earth and moon form, to a high degree of approximation, a two-particle Newtonian gravitational system" (Giere, 1988, 81). Aunque el modelo de Giere resulta fecundo para la reflexión metateórica en disciplinas como la economía o la sociología, es en la formulación de la hipótesis teórica donde los problemas propios de las ciencias sociales reaparecen. Como sostiene Amparo Gómez Rodríguez:

En ciencias sociales identificar elementos del modelo teórico con elementos del sistema social presenta, en algunos casos, cierto grado de dificultad. Un tema recurrente en economía es que los individuos no presentan la constancia en propiedades relevantes que supone la teoría ... Esto significa que la identificación de elementos del modelo teórico como elementos 
del sistema real se logra de forma parcial y en cierto grado. En otros casos, las dificultades para establecer la similaridad estructural son aún mayores, con lo cual la afirmación de la hipótesis teórica apenas es algo más que un recurso retórico (Gómez Rodríguez, 2003, 211-212).

La introducción del componente intencional en la reflexión sobre la ciencia es fundamental en la propuesta de Giere. Para él, los científicos utilizan los modelos para representar el mundo con distintos propósitos. Estos propósitos u objetivos pueden ser de índole teórica o de índole práctica. En "Economic Man as Model Man: Ideal Types, Idealization and Caricatures" Mary Morgan analiza la evolución del modelo de homo economicus desde Adam Smith a nuestros días pasando por William Jevons, Carl Menger, o Frank Knight entre otros. El retrato que elabora Mill, y señalábamos antes, es consistente con su definición de Economía Política. Jevons reduce las dimensiones de la utilidad de Bentham a solo dos, duración e intensidad, lo que le permite su representación en un eje de coordenadas. Knight lo dota con información completa y plena previsión del futuro, para que pudiera jugar el papel que le correspondía en la teoría económica completa. Morgan concluye: "These changes in characterization were associated with the long run changes in economic beliefs, theories, questions and practices" (2006, 27). Las necesidades de cada autor fueron diferentes y de la misma manera lo fueron sus modelos y esa es una caracterización de la modelización en ciencia que debemos a Giere.

Pero hay otro aspecto de la adecuación de los modelos a los propósitos de sus usuarios, que alejan a las ciencias sociales de las naturales, y las aproximan a las ciencias ingenieriles de las que hablamos en el apartado anterior. En ciencias sociales el objetivo no es solo obtener conocimiento acerca de cómo son y se comportan las cosas, sino posibilitar intervenciones en el mundo que modifiquen el curso de acción de determinados aspectos de la realidad social, como por ejemplo intervenciones en política fiscal o intervenciones socioeducativas. Esas intervenciones tienen que ser, igual que en el caso de los artefactos, eficientes y seguras. Por tanto, es sumamente importante que se analice cómo los datos que los investigadores seleccionan del mundo, para afirmar su adecuación al modelo, responden a los intereses teóricos, pero sobre todo prácticos de los científicos.

\section{Conclusión}

Aunque Giere no trató específicamente acerca de las ciencias ingenieriles ni de las sociales, su propuesta tiene un interesante recorrido para estas. Hemos destacado dos cuestiones en las que estas disciplinas guardan relaciones de similitud entre sí, y que, analizadas con las perspectivas clásicas, habían merecido un tratamiento que las minusvaloraba. Gracias a la propuesta de Giere, se puede apreciar que las diferencias con las disciplinas científicas tradicionales no son ni 
tan grandes ni tan relevantes. Estas dos cuestiones son, por un lado, la naturaleza y el papel que las leyes tienen en esos dominios y, por otro, los criterios de elección entre modelos.

Según la propuesta de Giere, todas las leyes están cualificadas de manera más o menos implícita con cláusulas ceteris paribus. En su ausencia, las leyes son enunciados altamente idealizados o, en el peor de los casos, directamente falsos. El hecho de que las leyes de las ciencias sociales e ingenieriles presentaran cláusulas ceteris paribus era una crítica que se les había hecho tradicionalmente, dado que se consideraba que era un problema que solo atañía a estas ciencias. Sin embargo, como Giere muestra, esto también se produce en las leyes más consolidadas y reconocidas de disciplinas que, como la física, eran consideradas el ejemplo a seguir. En la propuesta de Giere, las leyes serán los principios definitorios de los modelos y su verdad se establece dentro del modelo. Esto permite a las ciencias sociales salir de la eterna discusión acerca de la existencia o no de leyes en su seno $y$, por tanto, de su supuesta acientificidad y, en el caso de las ciencias ingenieriles, permite comprender mejor el hecho de que el carácter idealizado de estas leyes sea corregido en la práctica del diseño entre los ingenieros.

En segundo lugar, hemos enfatizado que la presencia de valores en la elección de modelos, tanto en ciencias ingenieriles como en ciencias sociales, se debe a que estas ciencias tienen entre sus objetivos prioritarios intervenir en el mundo. Una forma de plantearlo es considerar, en parte, a estas disciplinas como tecnologías, tanto artefactuales como sociales. En esa medida, es necesario introducir criterios de evaluación externos, que den cuenta de los posibles impactos sociales de las intervenciones en el mundo, que en ocasiones estos dominios de investigación plantean.

Desafortunadamente, la propuesta de Giere no ha sido suficientemente empleada en la reflexión sobre estas ciencias. Este pequeño repaso ha querido resaltar la utilidad de su enfoque, especialmente la idea de que la ciencia tiene características epistémicas especiales, pero sin que ello haya de conducirnos a las caracterizaciones idealizadas que se habían mantenido en la filosofía de la ciencia. Esto nos permite, a su vez, reivindicar el conocimiento social e ingenieril como un conocimiento científico en pie de igualdad con aquellas disciplinas que desde los enfoques más clásicos se habían considerado las auténticas ciencias.

\section{Referencias bibliográficas}

Gibson, Quentin (1982). La lógica de la investigación social. Madrid: Tecnos.

Giere, Ronald (1988). Explaining science: a Cognitive Approach. Chicago: University of Chicago Press.

Giere, Ronald (1999). Science without laws. Chicago: University of Chicago Press. 
Giere, Ronald (2004). How Models Are Used to Represent Reality. Philosophy of Science, 71, 742-752.

Gómez Rodríguez, Amparo (2001). Las leyes ceteris paribus y la inexactitud de la economía. Teorema, 20(3), 69-80.

Gómez Rodríguez, Aamparo (2003). Filosofía y metodología de las ciencias sociales. Madrid: Alianza.

Hobbes, Thomas (1651/1983). Leviatán. Madrid: Sarpe.

Mill, John Stuart (1836/1967). On the Definition of Political Economy; and on the Method of Philosophical Investigation in that Science. En J. S. Mill Essays on Economics and Society, editado por J. M. Robson, Collected Works of John Stuart Mill. Toronto: University of Toronto Press.

Morgan, Mary (2006). Economic Man as Model Man: Ideal Types, Idealization and Caricatures. Journal of the History of Economic Thought, 28(1), 1-27.

Moulines, C. Ulises (1982). Exploraciones metacientificas. Madrid: Alianza.

Roberts, John (2004). There are no laws of the social Science. En Christopher Hitchcock (ed.), Contemporary Debates in Philosophy of Science. New Jersey: Blackwell. 